\title{
Tuberculosis y odontología
}

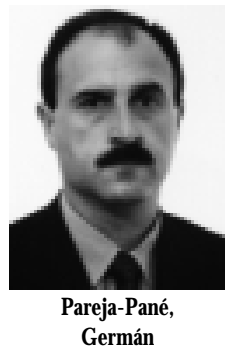

\author{
Tuberculosis and dentistry
}

Pareja- Pané, Germán

Profesor asociado. Unidad de Odontología Preventiva y Comunitaria. Facultad de Odontología. Universidad de Barcelona.

Resumen: se presenta en este artículo una revisión de los aspectos fundamentales de la tuberculosis y sus implicaciones en odontología. La tuberculosis, un importante problema de salud pública en la primera mitad del siglo pasado, disminuyó progresivamente su incidencia desde entonces a consecuencia de la mejoría en las condiciones de vida, las medidas de prevención y los progresos en el tratamiento. Sin embargo, la aparición de nuevas cepas de Mycobacterium tuberculosis resistentes a varios fármacos utilizados en el tratamiento y el aumento de su incidencia en inmunodeprimidos y otros grupos de población, agrava este problema. La tuberculosis se transmite, principalmente, por vía aérea. Cabe pues la posibilidad de que sea transmitida en el ejercicio de la profesión y aunque parece que el riesgo de transmisión es bajo, está indicado la introducción de medidas preventivas que reduzcan el riesgo.

Palabras clave: Tuberculosis, Mycobacterium tuberculosi, Odontología.

Abstract: In this paper, we present a review of the basic aspects of tuberculosis and its implications for dentistry. Tuberculosis, an important public health problem during the first half of the last century, has progressively decreased as a consequence of the improvement in the living conditions, preventive measures taken and better treatments. Nevertheless, the introduction of new multidrug-resistant strains of Mycobacterium tuberculosis and its increasing incidence among immunodeficient persons and other risk groups of the population have aggravated this problem. Tuberculosis is mainly transmitted by aerosols. Hence, it can be transmitted in dental settings, and, even though the risk of transmission is low, appropriate infection control strategies are needed.

Key words: Tuberculosis, Mycobacterium tuberculosis, Dentistry.

\section{Correspondencia}

Germán Pareja Pané

Odontología Preventiva y Comunitaria

Facultad de Odontología

Cl. Feixa Larga, $s / n$

08907 L'Hospitalet de Lobregat

(Barcelona)

\begin{tabular}{ccc}
\hline Fecha recepción & Fecha última revisión & Fecha aceptación \\
$05-05-2003$ & $22-12-2003$ & $12-01-2004$ \\
\hline
\end{tabular}

BIBபD [1138-123X (2004)9:3; mayo-junio 257-360]

Pareja-Pané G. Tuberculosis y odontología. RCOE 2004;9(3):327-332. 


\section{Introducción}

La tuberculosis es una enfermedad infecciosa que afecta principalmente a los pulmones y en menor medida a otros órganos como riñones, huesos, meninges y ganglios linfáticos entre otros. El microorganismo causante de la enfermedad es una micobacteria, Mycobacterium tuberculosis conocido también como bacilo tuberculoso o bacilo de Koch, en honor a su descubridor ${ }^{1 * *}$.

La tuberculosis, enfermedad conocida desde la antigüedad, constituyó a principios del siglo XX un importante problema de salud pública. En años posteriores, a consecuencia de la mejoría en las condiciones de vida, la puesta en marcha de medidas de prevención y a importantes progresos en su tratamiento, la incidencia de la enfermedad fue disminuyendo progresivamente llegándose a pensar que podría ser definitivamente erradicada. Sin embargo, el aumento de la incidencia de esta enfermedad en las últimas décadas muestra que la situación actual no es la que se esperaba²,3. Este problema se ha visto últimamente agravado por la aparición de cepas bacterianas resistentes a varios fármacos empleados en su tratamiento. En la actualidad, la tuberculosis, sin alcanzar los niveles de principios del siglo anterior, constituye un problema sanitario mundial de primer orden.

La vía de transmisión de la tuberculosis es aérea, por inhalación de partículas procedentes de secreciones respiratorias que contienen bacilos tuberculosos. Estas partículas proceden de enfermos que eliminan bacilos en sus secreciones respiratorias y que al toser, hablar o estornudar generan aerosoles, diminutas gotas que permanecen en suspensión en el aire y que son susceptibles de ser inhaladas por otros individuos, alcanzar los alvéolos pulmonares y transmitir la enfermedad. La vulnerabilidad del bacilo a la radiación ultravioleta hace difícil la transmisión en espacios abiertos o en locales iluminados por luz natural y bien ventilados. De hecho, los porcentajes más elevados de transmisión se encuentran entre los contactos próximos al enfermo que comparten su dormitorio (familiares, instituciones cerradas como cárceles, residencias de ancianos, hospitales para enfermos mentales, etc.). El estudio de contactos en el lugar de trabajo y en contactos ocasionales ha demostrado niveles muy bajos de transmisión ${ }^{1 * *}$.

La infección por Mycobacterium tuberculosis se produce cuando una persona susceptible inhala estas partículas en suspensión y los bacilos se introducen en el organismo a través de los alvéolos pulmonares. Gracias a la capacidad de la respuesta inmunológica del organismo humano, esta transmisión no tendrá consecuencias graves para el $90 \%$ de los afectados, que, a pesar de infectarse, no desarrollarán la enfermedad. Desgraciadamente, el $10 \%$ restante enfermará de tuberculosis en algún momento de su vida. En otras palabras, la transmisión e infección por el bacilo tuberculoso determina únicamente un riesgo de padecer la enfermedad pero no implica necesariamente su aparición.

La probabilidad de que una persona se infecte depende de la concentración de partículas en suspensión en el aire que contienen el bacilo y a su vez de factores ambientales y de las características del individuo fuente y su proceso patológico. Entre las causas ambientales cabe destacar el contacto entre individuos susceptibles y enfermos de tuberculosis en espacios pequeños y cerrados, una ventilación inadecuada que causa una menor dilución en el aire de estas partículas y una menor eliminación, y la recirculación del aire que las contiene. La mayor infectividad de un enfermo está relacionada con el número de microorganismos que expele al exterior y esto a su vez de la localización anatómica de la enfermedad, la presencia de tos o de otras maniobras que fuerzan al enfermo a lanzar al exterior secreciones con material contaminado, la capacidad del enfermo de taparse la boca cuando tose, la existencia de lesiones pulmonares cavitadas, la duración del tratamiento antituberculoso, etc. Los enfermos más infecciosos son aquellos que padecen tuberculosis pulmonar o laringea. Las localizaciones extrapulmonares comportan una menor infectividad excepto aquellas que se localizan en la cavidad oral o en el tracto respiratorio. Aunque no disponemos todavía de suficientes datos, parece que los enfermos de tuberculosis con síndrome de inmunodeficiencia adquirida tienen una infectividad similar a aquellos enfermos con tuberculosis que no padecen este síndrome. La infectividad es también mayor en aquellos enfermos con tos productiva y más si padecen lesiones pulmonares cavitadas. Es mayor la capacidad infectiva de un enfermo que no ha recibido tratamiento que la de quien ha recibido un tratamiento adecuado y en general cuanto mayor es el tiempo de tratamiento menor es la infectividad.

RCOE, 2004, Vol 9, №3, 327-332 


\section{Epidemiología}

La morbilidad y mortalidad de la enfermedad no son despreciables y, a pesar de la eficacia del tratamiento antituberculoso, la tuberculosis tiene consecuencias, en algunos casos graves, para quienes la padecen (tabla 1). En España, según datos del Centro Nacional de Epidemiología ${ }^{4}$, se notificaron en el año 2000 un total de 7.753 casos de tuberculosis pulmonar, lo que viene a representar una tasa de notificaciones de 19,64 casos por 100.000 habitantes $(22,67$ en 1998). Se observan diferencias importantes entre las diferentes comunidades autónomas, siendo Galicia $(49,88$ notificaciones por 100.000 habitantes en 1998, 40,13 notificaciones por 100.000 habitantes en el año 2000), Ceuta $(53,79$ notificaciones por 100.000 habitantes en 1998, 52,99 notificaciones por 100.000 habitantes en el año 2000) y Melilla $(69,14$ notificaciones por 100.000 habitantes en 1998, 56,56 notificaciones por 100.000 habitantes en el año 2000) las más afectadas (tabla 2).

La transmisión de la tuberculosis en las instalaciones sanitarias es un hecho conocido ${ }^{5,6}$. La magnitud de este riesgo depende del tipo de instalación sanitaria, de la población atendida, del tipo de intervención sanitaria que se lleva a cabo y de la categoría del personal sanitario. La transmisión nosocomial de la tuberculosis se asocia con el contacto próximo con pacientes tuberculosos y en procedimientos como la broncoscopia, la intubación endotraqueal y ventilación mecánica, la irrigación de abcesos abiertos y las autopsias. Los procedimientos que estimulan la tos y el tra-

\begin{tabular}{|cc|}
\hline $\begin{array}{c}\text { Tabla I. Defunciones por tubercu- } \\
\text { Iosis en España (1980- 1998) }\end{array}$ \\
\hline & \\
Año & Defunciones (N) \\
\hline 1980 & 1469 \\
\hline 1981 & 1558 \\
\hline 1982 & 1306 \\
\hline 1983 & 1228 \\
\hline 1984 & 1040 \\
\hline 1985 & 1059 \\
\hline 1986 & 965 \\
\hline 1987 & 939 \\
\hline 1988 & 896 \\
\hline 1989 & 804 \\
\hline 1990 & 861 \\
\hline 1991 & 791 \\
\hline 1992 & 765 \\
\hline 1993 & 720 \\
\hline 1994 & 634 \\
\hline 1995 & 603 \\
\hline 1996 & 601 \\
\hline 1997 & 523 \\
\hline 1998 & 476 \\
\hline Fuente: Instituto Nacional de Estadística \\
\hline
\end{tabular}

tamiento con aerosoles comportan también un mayor riesgo.

Ciertos procedimientos dentales como las preparaciones cavitarias con instrumental rotatorio especialmente a alta velocidad generan aerosoles detectables en el aire ambiental'. Cuando estos procedimientos se realizan en enfermos de tuberculosis cabe la posibilidad de que estas partículas en suspensión contengan bacilos tuberculosos que pueden infectar al personal sanitario. Parece, sin embargo, que el riesgo de transmisión al personal que trabaja en clínicas dentales es bajo, parecido al de la población general ${ }^{8-11}$. A pesar de ello es potencialmente posible y por este motivo está indicada la introducción de medidas preventivas que reduzcan el riesgo ${ }^{12^{* *}, 13}$.

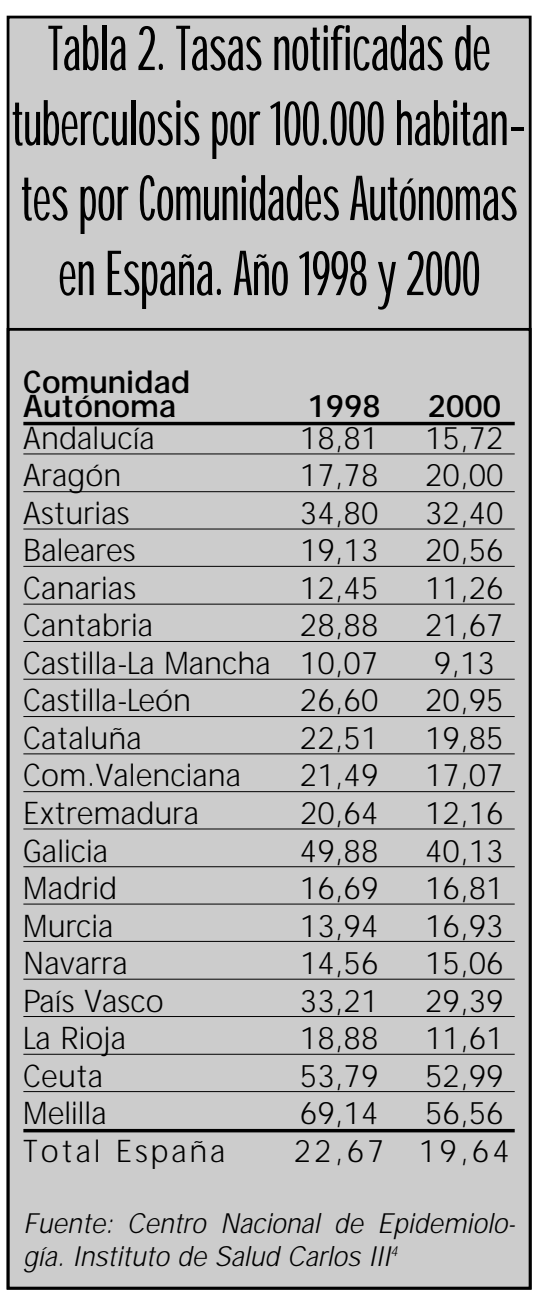

\section{Prevención}

La introducción de medidas preventivas en la clínica dental depende del nivel de riesgo de transmisión de la tuberculosis. En función de este nivel de riesgo se aplicarán las medidas preventivas más adecuadas. Es útil clasificar este riesgo en categorías (tabla 3) basándonos en diferentes factores. El primer factor es la incidencia de tuberculosis activa en el lugar geográfico en el que se desarrolla la actividad 
odontológica. La incidencia de tuberculosis activa en cada comunidad puede obtenerse de los datos epidemiológicos publicados por los diferentes organismos oficiales (en España en el Centro Nacional de Epidemiología ${ }^{3}$, organismo dependiente del Ministerio de Sanidad y Consumo). El segundo factor es el número de pacientes con tuberculosis activa que han sido visitados en la clínica dental en el último año. El tercer factor es la existencia o no de una posible transmisión anterior en la clínica dental (transmisión documentada entre pacientes o entre pacientes y profesional o entre profesional y paciente) $)^{12^{* *}}$. La mayoría de clínicas dentales pueden clasificarse en la categoría de mínimo riesgo o muy bajo riesgo.

Las medidas preventivas se clasifican para su comprensión en tres apartados:

\section{A) Prevenir la generación de micro- partículas en suspensión con mate- rial infeccioso.}

1. Identificación y tratamiento de los individuos con infección tuberculosa y con tuberculosis activa. La identificación de los individuos con infección tuberculosa, en ausencia de tuberculosis activa, se lleva a cabo con el test de Mantoux o de la tuberculina, que consiste en la inyección intradérmica de un derivado proteico purificado (PPD) y la observación de la presencia/ausencia de una reacción en el lugar de la inoculación. Un resultado positivo confirma la existencia de una infección presente o pasada por Mycobacterium tuberculosis. Este método diagnóstico no tiene una gran sensibilidad y especificidad y un resultado negativo no excluye la posibilidad de una infección tuberculosa o

\begin{tabular}{|ll|}
\hline Riesgo mínimo & Tabla 3. Categorías de riesgo \\
& $\begin{array}{l}\text { En la clínica dental no se ha atendido en el último año } \\
\text { ningún paciente con tuberculosis activa y la incidencia de } \\
\text { tuberculosis activa en la comunidad es baja. }\end{array}$ \\
\hline Riesgo muy bajo & $\begin{array}{l}\text { En la clínica dental no se ha atendido en el último año } \\
\text { ningún paciente con tuberculosis activa y la incidencia de } \\
\text { tuberculosis activa en la comunidad es media o alta. }\end{array}$ \\
\hline Riesgo bajo & $\begin{array}{l}\text { En la clínica dental se ha atendido a <6 pacientes con } \\
\text { tuberculosis activa aunque en ningún caso está } \\
\text { documentada la transmisión del bacilo tuberculoso. }\end{array}$ \\
\hline Riesgo medio & $\begin{array}{l}\text { En la clínica dental se ha atendido a }>5 \text { pacientes con } \\
\text { tuberculosis activa aunque en ningún caso está } \\
\text { documentada la transmisión del bacilo tuberculoso. }\end{array}$ \\
\hline Riesgo alto & $\begin{array}{l}\text { Está documentada la transmisión del bacilo tuberculoso en } \\
\text { la clínica dental (PPD* positivo en trabajador sanitario que } \\
\text { anteriormente era negativo, evidencia de transmisión entre } \\
\text { pacientes o entre pacientes y profesional o entre } \\
\text { profesional y pacientes) }\end{array}$ \\
\hline *derivado proteico purificado. \\
\hline
\end{tabular}

de una tuberculosis activa. Los falsos negativos se presentan con mayor frecuencia en las personas con infección por el virus de la inmunodeficiencia humana $(\mathrm{VIH})$.

El diagnóstico de tuberculosis activa se lleva a cabo mediante radiografías de tórax, examen microscópico del esputo en busca de bacilos y cultivo e identificación bacteriológica que es la prueba confirmatoria por excelencia ${ }^{1 * *}$.

El personal que trabaja en una clínica dental no es responsable del diagnóstico y tratamiento de la tuberculosis; sin embargo, debe conocer los aspectos básicos de la enfermedad que le permitan reconocer a los pacientes sospechosos de padecerla y referirlos al médico.

2. Determinación de la infectividad de los pacientes con tuberculosis. La capacidad infectiva de una persona con tuberculosis está en función del número de microorganismos que expulsa al exterior, lo cual a su vez depende, como se ha dicho anteriormente, de la localización anatómica de la enfermedad, la presencia de tos o de otras maniobras que fuerzan al enfermo a lanzar al exterior secreciones con material contaminado, la capacidad del enfermo de taparse la boca cuando tose, la existencia de lesiones pulmonares cavitadas y de la duración del tratamiento antituberculoso. La administración de un tratamiento antituberculoso adecuado reduce en gran manera la tos, la cantidad de esputo y el número de microorganismos, y en consecuencia la capacidad de transmitir la enfermedad. Sin embargo, el tiempo de tratamiento para que un paciente deje de ser bacilífero es variable y por ello el aislamiento preventivo del enfermo y la decisión de realizar ciertas intervenciones sanitarias como las dentales deben hacerse de un modo individualizado para cada paciente. En general, los pacientes con tuberculosis activa 
confirmada o sospechosos de padecerla deben ser considerados infecciosos cuando la tos es persistente o presentan una tinción o un cultivo positivos para Mycobacterium tuberculosis. Una persona en tratamiento durante unas 2-3 semanas que presenta una respuesta clínica y bacteriológica a este tratamiento (reducción de la tos, ausencia de fiebre y una disminución progresiva de la cantidad de bacilos en el esputo) puede ser considerada como no infecciosa ${ }^{1 * *}$.

3. Educación de los trabajadores sanitarios en:

- Los conceptos básicos de patogénesis y transmisión de Mycobacterium tuberculosis.

- La diferencia entre infección tuberculosa y tuberculosis activa.

- La sintomatología de la enfermedad.

- Las medidas de prevención.

B) Prevenir la diseminación de las micropartículas en suspensión con material infeccioso y reducir la contaminación microbiana en el aire.

1. Un método simple y efectivo de reducir la diseminación de estas micropartículas es el uso de mascarillas por los enfermos o la utilización de pañuelos que cubran la boca cuando tosen o estornudan.

2. Una adecuada ventilación de las áreas de tratamiento de estos pacientes diluye y elimina la cantidad de micropartículas en suspensión en el aire. Con ella aseguramos que el aire del local se renueve expulsándolo al exterior e introduciendo aire nuevo. Es importante que haya cierta presión negativa en el interior del local que impida que el aire acumulado salga a áreas vecinas. Adicionalmente y cuando no podemos renovar totalmente el aire es útil la utilización en los aparatos de ventilación de filtros que impidan el paso de partículas de pequeño tamaño. Con ello aseguramos que el aire recirculante no contenga o contenga una concentración menor de estas micropartículas en suspensión.

3. Utilización de lámparas ultravioletas. La radiación ultravioleta tiene capacidad germicida y por ello se ha propuesto la utilización de estas lámparas en el techo de los locales en los que se realizan intervenciones sanitarias o en los conductos por los que circula el aire para la ventilación. Su eficacia, sin embargo, no ha sido demostrada totalmente y hay que considerar que tienen efectos adversos: la sobreexposición a los rayos ultravioletas puede producir queratoconjuntivitis y eritema cutáneo.

\section{C) Protección del personal sanitario}

1. Utilización de mascarillas y otros protectores respiratorios. La utilización de mascarillas evita la inhalación de micropartículas en suspensión. Sin embargo, no protegen totalmente debido a su insuficiente sellado facial y a la posibilidad de que filtren partículas de un tamaño similar al de las micropartículas en suspensión. Esto último se agrava cuando la mascarilla está humedecida ${ }^{12^{* *}}$.

2. Manejo de los pacientes con tuberculosis activa. En general, debe evitarse tratar a los enfermos con tuberculosis activa, a excepción de los tratamientos de urgencia, hasta que hayan recibido tratamiento efectivo de su enfermedad y se reduzca o desparezca su capacidad de transmitir el bacilo.

\section{Conclusiones}

No parece que la tuberculosis se transmita con más frecuencia en las consultas dentales. El riesgo de infectarse del personal sanitario no es mayor que el de la población general ${ }^{8-11}$. No hay, sin embargo, demasiados estudios que confirmen o rebatan estos hechos y probablemente se necesita más investigación. Se impone la cautela y puesto que algunas intervenciones dentales son propicias a generar aerosoles que podrían contener material contaminado y por tanto existe posibilidad de transmisión, es necesaria la aplicación de medidas preventivas que la reduzcan o eliminen.

En general, se recomienda aplicar las medidas preventivas anteriormente mencionadas en todos los casos, con independencia de la categoría de riesgo en que se clasifiquen (tabla 3 ). En las categorías de riesgo media y alta se aconseja extremarlas y establecer protocolos para el examen periódico de los trabajadores sanitarios. 


\section{Bibliografía recomendada}

Para profundizar en la lectura de este tema, el/los autor/es considera/an interesantes los artículos que aparecen señalados del siguiente modo: *de interés $* *$ de especial interés.

1**. Dooley SW, Castro KG, Hutton MD, Mullan RJ, Polder JA, Snider DE Jr. Guidelines for preventing de transmission of tuberculosis in health-care settings, with special focus on HIV-related issues. MMWR Recomm Rep 1990;39(RR-17):1-29.

Excelente guía clínica respecto al riesgo de transmisión de la tuberculosis en el medio sanitario y su prevención, que refleja la opinión de los Centres for Control Disease and Prevention de los EEUU.

2. Tuberculosis rates and notification rates, 19961999 WHO European Region. (fecha de acceso 10 de junio de 2003). URL disponible en http://www.eurotb.org/eurotb.htm

3. Estadísticas de enfermedades de declaración obligatoria. Centro Nacional de Epidemiología. Instituto de Salud Carlos III. (fecha de acceso 10 de junio de 2003). URL disponible en http://193.146.50.130/
4. Mortalidad por tuberculosis en España por Comunidades Autónomas. Instituto Nacional de Estadística. (fecha de acceso 10 de junio de 2003(. URL disponible en http://www.ine.es

5. Barrett-Connor E. The epidemiology of tuberculosis in physicians. JAMA 1979;241(1):33-8.

6. Hutton MD, Stead WW, Cauthen GM, Bloch AB, Ewing WM. Nosocomial transmission of tuberculosis associated with a draining tuberculous abcess. J Infect Dis 1990;161(2): 286-95.

7. Bennett AM, Fulford MR, Walker JT, Bradshaw DJ, Martin MV, Marsh PD. Microbial aerosols in general dental practice. $\mathrm{Br}$ Dent $\mathrm{J}$ 2000;189(12):664-7.

8. Porteous NB, Brown JP. Tuberculin skin test conversion rate in health care workers. Results of a prospective study. Am J Infect Control 1999;27(5)385-7.

9. Anonymous. Tuberculosis in a dentist. Commun Dis Rep CDR Wkly 1999;9(29):253,256.
10. Mikitka D, Mills SE, Dazey SE, Gabriel ME Tuberculosis infection in US Air Force dentists. Am J Dent 1995;8(1):33-6.

11. Harlow, Rutkauskas JS. Tuberculosis risk in the hospital dental practice. Spec Care Dentist 1995;15 (2):50-5.

12**. Cleveland JL, Gooch BF, Bolyard EA, Simone PM, Mullan RJ, Marianos DW. Tuberculosis infection recommendations from the CDC, 1994.: considerations for dentistry. United States Centers for Disease Control and Prevention. J Am Dent Assoc 1995;126(5) 593-9.

Resumen del artículo de Dooley, Castro y cols con especial énfasis en el medio dental.

13. Cleveland JL, Gooch BF, Valway SE, Marianos DW, Butler WR, Onorato IM. Multidrug-resistant Mycobacterium tuberculosis in an HIV dental clinic. Infect Control Hosp Epidemiol 1995;16(1):7-11. 